\title{
Comparison of Methods for the Identification of Coagulase- negative Staphylococci
}

\author{
Maria de Lourdes RS Cunha/ ${ }^{+}$, Yuri K Sinzato, Liciana VA Silveira*
}

\author{
Departamento de Microbiologia e Imunologia *Departamento de Bioestatística, Instituto de Biociências, Universidade \\ Estadual Paulista, 18618-000 Botucatu, SP, Brasil
}

Coagulase-negative staphylococci (CNS) species identification is still difficult for most clinical laboratories. The scheme proposed by Kloos and Schleifer and modified by Bannerman is the reference method used for the identification of staphylococcal species and subspecies; however, this method is relatively laborious for routine use since it requires the utilization of a large number of biochemical tests. The objective of the present study was to compare four methods, i.e., the reference method, the API Staph system (bioMérieux) and two methods modified from the reference method in our laboratory (simplified method and disk method), in the identification of 100 CNS strains. Compared to the reference method, the simplified method and disk method correctly identified 100 and $99 \%$ of the CNS species, respectively, while this rate was $84 \%$ for the API Staph system. Inaccurate identification by the API Staph method was observed for Staphylococcus epidermidis (2.2\%), S. hominis (25\%), S. haemolyticus (37.5\%), and $\mathrm{S}$. warneri (47.1\%). The simplified method using the simple identification scheme proposed in the present study was found to be efficient for all strains tested, with $100 \%$ sensitivity and specificity and proved to be available alternative for the identification of staphylococci, offering, higher reliability and lower cost than the currently available commercial systems. This method would be very useful in clinical microbiology laboratory, especially in places with limited resources.

Key words: coagulase-negative staphylococci - methods - identification - API Staph

Forty species of the genus Staphylococcus have been identified thus far (Trülzsch et al. 2002, Bannerman 2003, Kwok \& Chow 2003, Spergser et al. 2003). S. aureus, a coagulase-positive species which produces a series of other enzymes and toxins, is the best known and has been frequently implicated in the etiology of a series of infections and intoxications in animals and humans, whereas coagulase-negative staphylococci (CNS), representing the majority of species, have been considered to be saprophytic or rarely pathogenic (Kloos \& Schleifer 1975). Over the last decade, however, CNS have been recognized as the etiological agents of a series of infectious processes, representing the microorganisms most commonly isolated from blood cultures (Huebner \& Goldmann 1999).

About half of CNS species naturally colonize humans, and at present they are considered essentially opportunistic etiological agents, that prevail in numerous organic situations to produce severe infections (Bannerman 2003). The emergence of CNS as pathogens of different infections can be the result of the increasing use of invasive procedures such as intravascular catheters and prostheses in patients undergoing intensive treatment, immunocompromised patients, premature children, patients with neoplasias, and transplant patients (Kloos \& Bannerman 1994).

${ }^{+}$Corresponding author. Fax: +55-14-3815.3744. E-mail: cunhamlr@ibb.unesp.br

Received 30 August 2004

Accepted 5 Novembert 2004
The species that most frequently cause diseases in humans are $S$. epidermidis (bacteremia, infections due to implanted medical devices such as prostheses and catheters, infection of surgical wounds, peritonitis in patients on continuous peritoneal dialysis, osteomyelitis, endophthalmitis etc.), S. haemolyticus (endocarditis, peritonitis, septicemia, and infections of the urinary tract, wounds, bone, and joints), and S. saprophyticus (urinary infections and septicemic processes). Other significant opportunistic pathogens include $S$. hominis, S. warneri, S. capitis, S. simulans, S. cohnii, S. xylosus, and S. saccharolyticus (Bannerman 2003). S. lugdunensis seems to be associated with endocarditis after implantation of prosthetic valves, with peritonitis, with soft tissue infection, and with vertebral osteomyelitis (Osmon et al. 2000).

In view of the known pathogenic potential of $\mathrm{CNS}$ within the hospital environment, interest regarding the variety of species related to infection and their toxigenic potential and virulence has increased over the last decade and has led to the publication of various studies on these aspects. However, despite the growing characterization of CNS infections, these microorganisms are not identified in clinical microbiology laboratories. The scheme proposed by Kloos and Schleifer (1975) and modified by Bannerman (2003) is the method conventionally used; however, this method is relatively laborious for routine use since a large number of biochemical tests are required. In most laboratories of routine, staphylococci are identified based on morphological aspects of the colonies, gram staining and catalase and coagulase production, which only permit the classification of staphylococci into $S$. aureus and non-S. aureus isolates, with the latter simply being classified as $\mathrm{CNS}$. 
The development of methods for the identification of staphylococcal species and subspecies permits clinicians to obtain information about the variety of CNS present in clinical specimens and to consider them as etiological agents of infectious processes. Accurate identification of CNS is needed in order to have an early prediction of the potential pathogenicity or antibiotic susceptibility of each clinical isolate and to clarify the clinical significance of each species. Repeat CNS isolates from patients with invasive diseases should be identified to allow a comparison of the strains. On the other hand, species identification is a prerequisite before typing procedures for epidemiological studies are undertaken.

In recent years, several commercial systems for the rapid identification of staphylococci have been developed as an alternative to the classical identification protocols (Bannerman 2003). However, these diagnostic systems present problems such as cost and incubation time, often provide unreliable results (Grant et al. 1994, Perl et al. 1994). Additionally, many of these kits were designed for the identification of all known CNS species (i.e., clinical, veterinary, and alimentary isolates) and thus are not very specific. Based on the above considerations and in view of the need for rapid, simple, and reliable methods, the objective of the present study was to compare four techniques for the identification of CNS, i.e., a reference method (Kloos \& Schleifer 1975, Bannerman 2003), the commercial API Staph system, and two methods modified from the reference method in our laboratory to develop alternative identification methods combining simplicity, reliability, and low cost, especially to places with limited resources.

\section{MATERIALS AND METHODS}

Isolates - One-hundred CNS isolates obtained from clinical specimens of patients hospitalized at the University Hospital of the Faculty of Medicine, Universidade Estadual Paulista (Unesp), Botucatu Campus, were studied. Strains were isolated as described by Koneman et al. (1997).

Identification of CNS - The isolates obtained from clinical specimens were plated onto blood agar and gram stained in order to guarantee their purity and the preservation of their morphology and specific staining. After confirmation of these characteristics, the isolates were submitted to the catalase and coagulase tests. Staphylococcus was differentiated from Micrococcus species on the basis of the oxidation and fermentation of glucose, resistance to bacitracin $(0.04 \mathrm{U})$ indicated by absence of an inhibition halo or presence of an inhibition halo measuring up to $9 \mathrm{~mm}$ in diameter, and susceptibility to furazolidone $(100 \mu \mathrm{g})$ characterized by inhibition zones measuring 15 to $35 \mathrm{~mm}$ in diameter (Baker 1984).

The four methods described below were used for the identification of CNS. The following international reference CNS strains were used as controls: S. epidermidis (ATCC 12228), S. simulans (ATCC 27851), S. warneri (ATCC 10209), S. xylosus (ATCC 29979), and $S$. saprophyticus (ATCC 15305).

Reference method proposed by Kloos and Schleifer (1975) and Bannerman (2003) - This method consists of a set of biochemical tests that determine the utilization of the sugars xylose, arabinose, sucrose, trehalose, maltose, mannitol, lactose, xylitol, ribose, fructose, and mannose, production of hemolysin, nitrate reduction, presence of urease and ornithine decarboxylase, and resistance to novobiocin characterized by an inhibition halo of up to $16 \mathrm{~mm}$. Readings of the tests were obtained after 24,48 , and $72 \mathrm{~h}$ of incubation at $37^{\circ} \mathrm{C}$ in an air incubator.

API Staph - The API Staph system (bioMérieux) is a ready-to-use test battery consisting of 20 biochemical tests to which an homogenous bacterial suspension at $0.5 \mathrm{McF}$ arland turbidity is added. After $24 \mathrm{~h}$ of incubation at $37^{\circ} \mathrm{C}$ and addition of the VP (VP1 and VP2), NIT (NIT1 and NIT2), and PAL (ZYM A and ZYM B) reagents accompanying the kit, the reactions were interpreted and microorganisms were identified using the analytical cata$\log$. Identification is based on a numerical system consisting of seven digits which provides percent identification (\%ID), with a value $\geq 80 \%$ being acceptable.

Modified methods - Two identification methods modified in our laboratory were used (simplified method and disk method). The simplified method was divided into two steps. During the first step, fermentation of xylose, sucrose, trehalose, maltose, and mannitol, production of hemolysin, and anaerobic growth in thioglycolate were tested (Table I). The tests used in the second step varied according to the results obtained in the first identification step after $72-\mathrm{h}$ incubation at $37^{\circ} \mathrm{C}$. The complementary tests used during the second step (when necessary) are specified in Table II.

The disk method consisted of the following tests: fermentation of arabinose, sucrose, trehalose, maltose, mannitol and lactose, nitrate reduction, production of hemolysin, tests for urease and ornithine decarboxylase, and resistance to novobiocin. For the sugar fermentation test, commercially available disks specific for each sugar were placed in tubes containing $2.5 \mathrm{ml}$ Purple Broth Base medium. Bacterial suspensions were inoculated as described by Kloos and Schleifer (1975). Readings for the two methods were obtained after 24,48 , and $72 \mathrm{~h}$ of incubation at $37^{\circ} \mathrm{C}$, and CNS species were identified according to the identification scheme proposed in the Figure.

Statistical analysis - To determine the degree of agreement between the methods used for the identification of CNS (simplified method, disk method and API Staph) and the reference method (Kloos \& Schleifer 1975, Bannerman 2003), the sensitivity and specificity of the tests (Sox 1986) were assessed as follows.

Sensitivity: proportion of CNS strains that tested positive for a certain species by the reference method and which were identified as the same species by the method analyzed (simplified method, disk or API Staph).

Specificity: proportion of CNS strains that tested negative for a certain species by the reference method and which were also negative for the same species when tested by the method analyzed (simplified method, disk or API Staph).

\section{RESULTS}

The 100 staphylococcal isolates were tested by the four proposed methods. The results obtained with the reference method (Kloos \& Schleifer 1975, Bannerman 
TABLE I

Summary of the tests used in the first step of the simplified method for the identification of human Staphylococcus species

Species

Coagulase D-Xylose Sucrose D-Trehalose Maltose Manitol Anaerobic Hemolysis growth thioglycolate

\begin{tabular}{|c|c|c|c|c|c|c|c|c|}
\hline S. aureus ${ }^{a}$ & + & - & + & + & + & + & + & + \\
\hline S. schleiferi subsp. coagulans ${ }^{a}$ & + & - &,+- & - & - &,+- & + & + \\
\hline S. schleiferi subsp. schleiferi ${ }^{b}$ & - & - & - &,+- & - & - & + & + \\
\hline S. epidermidis ${ }^{a}$ & - & - & + & - & + & - & + &,+- \\
\hline S. haemolyticus ${ }^{b}$ & - & - & + & + & + &,+- & + & + \\
\hline S. saprophyticus $b$ & - & - & + & + & + &,+- & + & - \\
\hline S. warneri ${ }^{b}$ & - & - & + & + & + &,+- & + &,+- \\
\hline S. hominis subsp. hominis ${ }^{a}$ & - & - & + &,+- & + & - & - & - \\
\hline S. hominis subsp. novobiosepticus ${ }^{b}$ & - & - & + & - & + & - & - & - \\
\hline S. simulans ${ }^{a}$ & - & - & + &,+- & \pm & + & + &,+- \\
\hline S. lugdunensis $b$ & - & - & + & + & + & - & + & + \\
\hline S. capitis subsp. capitis ${ }^{a}$ & - & - & + & - & - & + & + &,+- \\
\hline S. capitis subsp. urealyticus ${ }^{a}$ & - & - & + & - & + & + & + &,+- \\
\hline S. cohnii subsp. cohnii ${ }^{b}$ & - & - & - & + &,+- &,+- & + &,+- \\
\hline S. cohnii subsp. urealyticum ${ }^{b}$ & - & - & - & + & + & + & + &,+- \\
\hline S. xylosus ${ }^{a}$ & - &,+ \pm & + & + & + & + &,+- & - \\
\hline S. caprae ${ }^{b}$ & - & - & - & + &,+- &,+- & + &,+- \\
\hline
\end{tabular}

+: positive reaction; -: negative reaction; +,-: positive or negative; $a$ : species identified in a single step; $b$ : species identified in two steps.

\section{TABLE II}

Biochemical tests used in the second identification step of the simplified method for the identification of human Staphylococcus species

Result of 1 st step sucrosē (-)

Nitrate reductionUrease production

S. cohnii subsp. cohnii

S. cohnii subsp. urealyticum

S. caprae

S. schleiferi subsp. schleiferi

$\begin{array}{ll}- & - \\ - & + \\ + & + \\ + & -\end{array}$

Result of 1 st step sucrose (+) mannitol (-) hemolysis (+)

$\beta$-D-fructose $\begin{gathered}\text { Urease } \\ \text { production }\end{gathered} \begin{gathered}\text { Ornithine } \\ \text { decarboxylase }\end{gathered}$

S. warneri

S. haemolyticus

S. lugdunensis

+
,+-
+

$+$

$\begin{array}{llll}\text { S. lugdunensis } & + & +,- & + \\ \text { Result of 1st step }\end{array}$

sucrose (+) mannitol (-) hemolysis (-)

Resistance to novobiocin

S. warneri

S. saprophyticus

$+$

\begin{tabular}{lccc}
\hline \multicolumn{4}{c}{ Result of 1st step } \\
& \multicolumn{4}{c}{$\begin{array}{l}\text { sucrose (+) mannitol (+) } \\
\text { S-D-fructose }\end{array}$} & $\begin{array}{c}\text { Urease } \\
\text { production }\end{array}$ & $\begin{array}{c}\text { Resistance to } \\
\text { novobiocin }\end{array}$ \\
S. saprophyticus & + & + & + \\
S. waemori & + & + & - \\
\hline
\end{tabular}

+ +: positive reaction; -: negative reaction;,+- : positive or negative
2003) were compared to those obtained with the modified methods and the API Staph system.

Table III shows the agreement in identification between the analyzed assays and the reference method. The simplified method and the disk method showed 100 and $99 \%$ positivity when compared to the reference method, while this percentage was $84 \%$ for the API Staph system. Of the 16 isolates with disagreement of identification by the API Staph method, $10(62.5 \%)$ showed correct identification but with a \% ID of 7.1 to $31 \%$, lower than the acceptable value.

The international reference strains (ATCC) of $S$. epidermidis, S. simulans, S. saprophyticus, and S. xylosus were correctly identified by the four methods. Only the $S$. warneri reference strain (ATCC 10209) was inaccurately identified by the API Staph system, with characterization of this strain as $S$. saprophyticus $(\% \mathrm{ID}=58.7 \%)$, as $S$. hominis $(\% \mathrm{ID}=19.5 \%)$, or as $S$. warneri $(\% \mathrm{ID}=15.8 \%)$.

The simplified method carried out in two steps did not differ from the reference method in terms of the identification of CNS species. In contrast to the other methods, the disk method showed inaccurate identification and erroneously identified an $S$. hominis strain $(6.5 \%)$ due to the non-fermentation of sucrose on the disk, leading to the classification of this strain as $S$. caprae. No incongruence was observed for the other species.

Inaccurate identification by the API Staph system was observed for S. epidermidis (2.2\%), S. warneri (47.1\%), S. hominis (25\%), and S. haemolyticus (37.5\%), while agreement with the reference method was $100 \%$ for $S$. lugdunensis, S. simulans, S. xylosus, S. saprophyticus, $S$. caprae, and $S$. cohnii subspecies urealyticum.

The largest discrepancy was observed between the reference method and the API Staph system, with the latter method not accurately identifying $1 \mathrm{~S}$. epidermidis 
strain (identified as $S$. lugdunensis), 3 S. haemolyticus strains (2 identified as $S$. aureus and 1 as $S$. hominis), $4 S$. hominis strains (2 identified as $S$. lugdunensis, 1 as $S$. haemolyticus and 1 as $S$. aureus), and $8 S$. warneri strain (3 identified as S. lugdunensis, 2 as S. haemolyticus, 2 as S. hominis and 1 as $S$. saprophyticus) (Table III).

As shown in Table IV, the sensitivity and specificity of the simplified method were $100 \%$ for all species studied. The disk method showed $93.8 \%$ sensitivity for the identification of S. hominis and $100 \%$ sensitivity for the other species, while specificity was $98.8 \%$ for S. hominis, $98.9 \%$ for $S$. caprae, and $100 \%$ for the other species. The sensitivity of the API Staph was $100 \%$ for S. lugdunensis, S. simulans, S. xylosus, S. saprophyticus, S. caprae, and S. cohnii subspecies urealyticum, $97.8 \%$ for $S$. epidermidis, $52.9 \%$ for $S$. warneri, $75 \%$ for S. hominis, and $62.5 \%$ for $S$. haemolyticus. The specificity of this test ranged from 94.8 to $98.9 \%$ for $S$. warneri, S. hominis, $S$. haemolyticus, S. lugdunensis, and S. saprophyticus strains, and was $100 \%$ for the other species.

\section{DISCUSSION}

CNS are the microorganisms most commonly isolated from blood cultures, representing a serious health problem in many developing countries and also developed (Renneberg et al. 1995). Some studies have suggested an association between $S$. epidermidis and nosocomial infections (Vuong \& Otto 2002) with this species being identified in 74 to $92 \%$ of patients with bacteremias caused by CNS (Martin et al. 1989). However, other studies have reported a series of infections caused by other CNS species (Herwaldt et al. 1996), mainly S. haemolyticus which

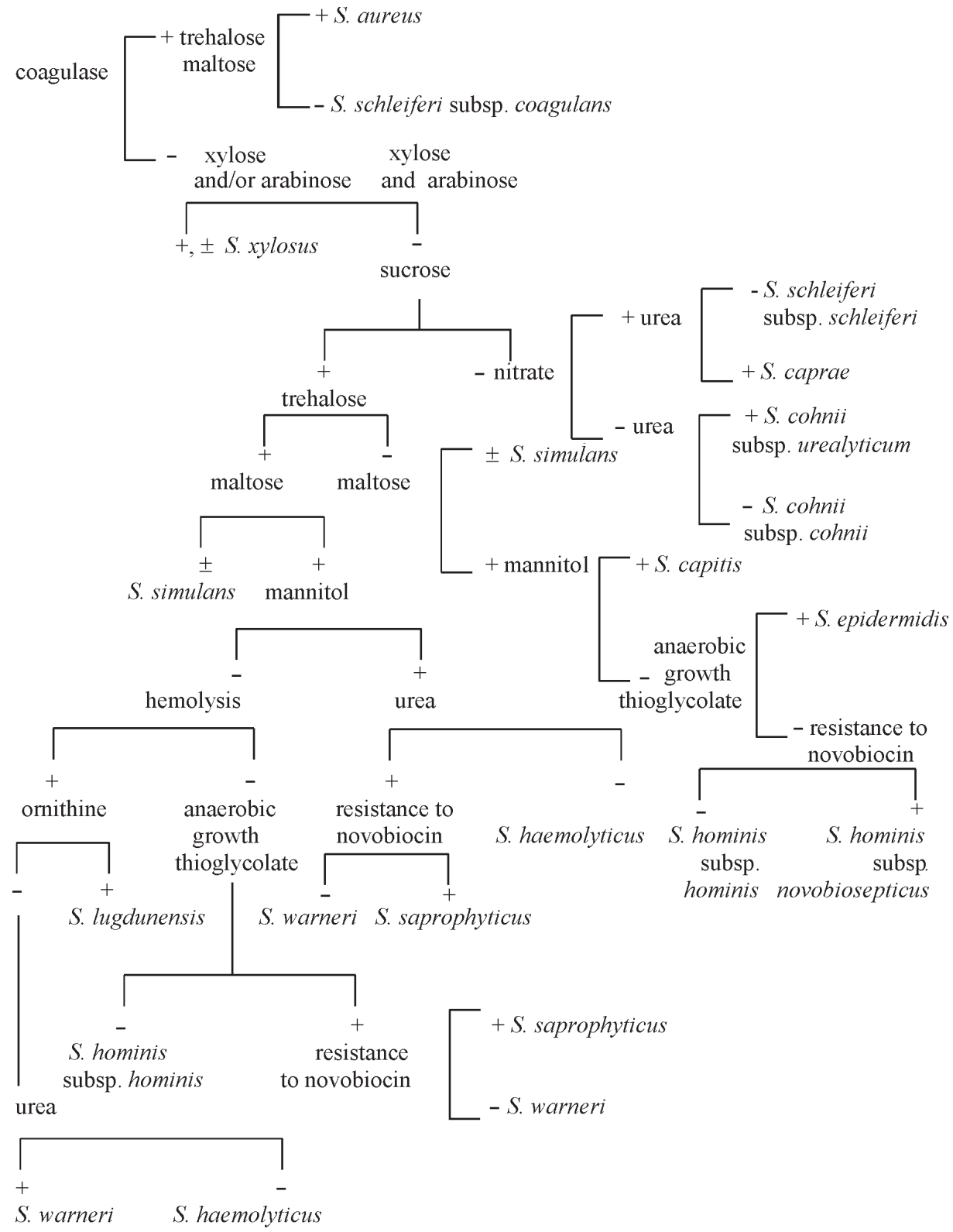

Simple scheme for the identification of human Staphylococcus species; +: positive; -: negative; +, -: positive or negative; \pm : weak 
TABLE III

Identification agreement between the modified methods and the API Staph system compared to the reference method

\begin{tabular}{|c|c|c|c|c|c|c|c|}
\hline \multirow{3}{*}{ Species } & \multirow{3}{*}{$\begin{array}{c}\mathrm{Nr} \text { of isolates tested } \\
\mathrm{N}\end{array}$} & \multicolumn{6}{|c|}{ Methods } \\
\hline & & \multicolumn{2}{|c|}{ Simplified } & \multicolumn{2}{|c|}{ Disk } & \multicolumn{2}{|c|}{ API Staph } \\
\hline & & $\mathrm{N}$ & $\%$ & $\mathrm{~N}$ & $\%$ & $\mathrm{~N}$ & $\%$ \\
\hline S. epidermidis & 45 & 45 & 100 & 45 & 100 & 44 & 97,8 \\
\hline S. warneri & 17 & 17 & 100 & 17 & 100 & 9 & 52,9 \\
\hline S. hominis & 16 & 16 & 100 & 15 & 93,8 & 12 & 75 \\
\hline S. haemolyticus & 8 & 8 & 100 & 8 & 100 & 5 & 62,5 \\
\hline S. lugdunensis & 4 & 4 & 100 & 4 & 100 & 4 & 100 \\
\hline S. simulans & 3 & 3 & 100 & 3 & 100 & 3 & 100 \\
\hline S. xylosus & 2 & 2 & 100 & 2 & 100 & 2 & 100 \\
\hline S. saprophyticus & 2 & 2 & 100 & 2 & 100 & 2 & 100 \\
\hline S. caprae & 2 & 2 & 100 & 2 & 100 & 2 & 100 \\
\hline S. cohnii & 1 & 1 & 100 & 1 & 100 & 1 & 100 \\
\hline Totals & 100 & 100 & 100 & 99 & 99 & 84 & 84 \\
\hline
\end{tabular}

S: Staphylococcus

TABLE IV

Sensitivity and specificity of the simplified method, disk method, and API Staph system for the identification of human Staphylococcus species compared to the reference method ${ }^{a}$

\begin{tabular}{|c|c|c|c|c|c|c|}
\hline \multirow[b]{2}{*}{ Species } & \multicolumn{3}{|c|}{ Sensitivity (\%) } & \multicolumn{3}{|c|}{ Specificity (\%) } \\
\hline & Simplified & Disk & API Staph & Simplified & Disk & API Staph \\
\hline S. epidermidis & 100 & 100 & 97,8 & 100 & 100 & 100 \\
\hline S. warneri & 100 & 100 & 52,9 & 100 & 100 & 98,8 \\
\hline S. hominis & 100 & 93,8 & 75 & 100 & 98,8 & 97,6 \\
\hline S. haemolyticus & 100 & 100 & 62,5 & 100 & 100 & 96,7 \\
\hline S. lugdunensis & 100 & 100 & 100 & 100 & 100 & 94,8 \\
\hline S. simulans & 100 & 100 & 100 & 100 & 100 & 100 \\
\hline S. xylosus & 100 & 100 & 100 & 100 & 100 & 100 \\
\hline S. saprophyticus & 100 & 100 & 100 & 100 & 100 & 98,9 \\
\hline S. caprae & 100 & 100 & 100 & 100 & 98,9 & 100 \\
\hline S. cohnii & 100 & 100 & 100 & 100 & 100 & 100 \\
\hline
\end{tabular}

$a$ : Gold Standard ( Kloos \& Schleifer 1975, Bannerman 2003)

is the second most frequently detected species (Bannerman 2003). Since CNS are the etiological agents of a series of infectious processes, identification of these microorganisms is important for the determination of their physiopathological characteristics and clinical importance and for epidemiological studies, and has led to the publication of various studies analyzing identification methods for these bacteria (Knapp \& Washington 1989, Bannerman et al. 1993, Piccolomini et al. 1994, Renneberg et al. 1995, Ieven 1995, De Paulis et al. 2003).

In the present study, the methods modified in our laboratory yielded good results in terms of the correct classification of CNS species compared to the reference method, with $100 \%$ agreement being observed for the simplified modified method and $99 \%$ agreement for the disk method.

The simplified method using the identification scheme proposed here (Figure) led to the identification of $S$. epidermidis, S. hominis, S. xylosus, S. capitis, and $S$. simulans in a single step, using a total of seven biochemical tests, a number lower than that employed in the reference method (16 tests). Since $S$. epidermidis is the most frequently isolated species, 70 to $90 \%$ of the strains
(Bannerman 2003) isolated in the clinical laboratory can be identified using a reduced number of tests.

With respect to incubation time, the results showed that $91 \%$ of the strains analyzed in the study fermented the species-specific sugar within $48 \mathrm{~h}$ of incubation at $37^{\circ} \mathrm{C}$. The other strains $(9 \%)$ tested positive for the fermentation of given sugars after $72 \mathrm{~h}$ of incubation, demonstrating the importance of an incubation of the sugar fermentation tests of at least $72 \mathrm{~h}$ in order to correctly identify these microorganism.

The identification of S. cohnii, S. schleiferi subspecies schleiferi, S. caprae, S. warneri, S. haemolyticus, $S$. saprophyticus, and $S$. lugdunensis required the execution of two or three additional biochemical tests, referred to as the second step, which varied according to the result obtained in the first step of simplified method. However, $20(37.7 \%)$ strains that required the second step for their identification fermented trehalose within $24 \mathrm{~h}$, thus permitting prior continuation of the additional tests. A longer time was needed to identify $S$. cohnii, S. schleiferi subspecies schleiferi, and $S$. caprae, since the second step required for the identification of these species in- 
cluded the nitrate reduction test whose result is only available after $48 \mathrm{~h}$. However, this fact does not actually result in a delay in the diagnosis of CNS since the frequency of these species in clinical samples is low.

The disk method was also found to be highly efficient and practical since it does not require previous preparation of sugars, thus preventing the loss of culture media, in addition to reaching high agreement in the identification of CNS with the reference method.

The commercial API Staph kit showed the lowest accuracy in the identification of CNS among the methods studied (84\% agreement), in agreement with the studies of (Bannerman et al. 1993, Renneberg et al. 1995).

$S$. warneri and S. hominis were the most difficult species to identify. Bannerman et al. (1993) also reported a lower accuracy in the identification of these species. In the study of Ieven et al. (1995), S. hominis was identified with the least accuracy by the API ID 32 Staph system. This finding might be explained by the lack of complementary tests such as novobiocin resistance, anaerobic growth in thioglycolate and hemolysin production.

In the case of S. haemolyticus, incorrect identification by the API Staph system might be explained by the fact that the kit does not suggest hemolysin production as a complementary test, which would be essential for the identification of $S$. haemolyticus strains.

Three $(3 \%)$ of the 100 strains analyzed by the API Staph system were identified as $S$. aureus, a fact also reported by Renneberg et al. (1995). The kit was found to be inefficient in these cases since it did not request the result of the fundamental and most widely accepted test for the identification of $S$. aureus, i.e., the coagulase test (Koneman et al. 1997).

According to Piccolomini et al. (1994), the low agreement between the API Staph and the traditional biochemical test for identification of CNS can be explained by the use of different incubation times, substrate concentrations and/or sensitivity markers.

In conclusion, the two methods modified in our laboratory were found to be highly efficient for routine use due to their high sensitivity and specificity compared to the reference method, in addition to requiring fewer tests and thus being more economical and faster than the standard method. Despite requiring a shorter incubation time $(18 \mathrm{~h})$, the API Staph system showed a lower sensitivity in the identification of some species. Undoubtedly, CNS species identification will be facilited and encouraged by the availability of a simple, inexpensive, and accurate procedure, especially in places with limited resources.

\section{ACKNOWLEDGMENTS}

To bioMérieux for the donation of the API Staph kits used in the present study.

\section{REFERENCES}

Baker JS 1984. Comparison of various methods for differentiation of staphylococci and micrococci. J Clin Microbiol 19: 875-879.

Bannerman TL 2003. Staphylococcus, Micrococcus, and other catalase-positive cocci that grow aerobically. In PR Murray, EJ Baron, JH Jorgensen, MA Pfaller, RH Yolken (eds), Manual of Clinical Microbiology, American Society Microbiology, Washington, p. 384-404.
Bannerman TL, Kleeman KT, Kloos WE 1993. Evaluation of the Vitek Systems Gram-positive identification card for species identification of coagulase-negative staphylococci. J Clin Microbiol 31: 1322-1325.

De Paulis AN, Predari S, Chazarreta CD, Santoianni JE 2003. Five-test simple scheme for species-level identification of clinically significant coagulase-negative staphylococci. J Clin Microbiol 41: 1219-1224.

Grant CE, Sewell DL, Pfaller M, Bumgardner RVS, Willians JA 1994. Evaluation of two commercial systems for identification of coagulase-negative Staphylococcus to species level. Diag Microbiol Infect Dis 18: 1-5.

Herwaldt LA, Geiss M, Kao C, Pfaller M 1996. The positive value of isolating coagulase-negative staphylococci from blood cultures. Clin Infect Dis 22: 14-20.

Huebner J, Goldmann DA 1999. Coagulase-negative staphylococci: role as pathogens. Annu Rev Med 50: 223-236.

Ieven M, Verhoeven J, Pattyn SR, Goossens H 1995. Rapid and economical method for species identification of clinically significant coagulase-negative staphylococci. J Clin Microbiol 33: 1060-1063.

Kloos WE, Bannerman TL 1994. Update on clinical significance of coagulase-negative staphylococci. Clin Microbiol Rev 7: 117-140.

Kloos WE, Schleifer KH 1975. Simplified scheme for routine identification of human Staphylococcus species. J Clin Microbiol 1: 82-88.

Knapp CC, Washington JA 1989. Evaluation of trehalose-mannitol broth for differentiation of Staphylococcus epidermidis from other coagulase-negative staphylococcal species. J Clin Microbiol 27: 2624-2626.

Koneman EW, Allen SD, Janda WM, Schreckenberger PC, Winn Jr WC 1997. Color Atlas and Textbook of Diagnostic Microbiology, 5th ed., Lippincott, Philadelphia, 1395 pp.

Kwok AYC, Chow AW 2003. Phylogenetic study of Staphylococcus and Macrococcus species based on partial hsp60 gene sequences. Int J Syst Evol Microbiol 53: 87-92.

Martin MA, Pfaller MA, Wenzel RP 1989. Coagulase-negative staphylococcal bacteremia. Ann Intern Med 110: 9-16.

Osmon DR, Sampathkumar P, Cockerill FR 2000. Prosthetic joint infection due to Staphylococcus lugdunensis. Mayo Clinic Proceedings 75: 511-512.

Perl TM, Rhomberg PR, Bale MJ, Fuchs PC, Jones RN, Koontz FP, Pfaller MA 1994. Comparison of identification systems for Staphylococcus epidermidis and other coagulasenegative Staphylococcus species. Diag Microbiol Infect Dis 18: 151-155.

Piccolomini R, Catamo G, Picciani C, D’Antonio D 1994. Evaluation of Staph-System 18-R for identification of staphylococcal clinical isolates to the species level. J Clin Microbiol 32: 649-653.

Renneberg J, Rieneck K, Gutschik E 1995. Evaluation of Staph ID system and Staph Zym system for identification of coagulase-negative staphylococci. J Clin Microbiol 33: 1150-1153.

Spergser J, Wieser, M, Täubel M, Rosselló-Mora RA, Rosengarten R, Busse HJ 2003. Staphylococcus nepalensis sp. nov., isolated from goats of the Himalayan region. Int J Syst Evol Microbiol 53: 2007-2011.

Sox HC 1986. Probability theory in the use of diagnostic tests. An introduction to critical study of literature. Ann Intern Med 104: 60-66.

Trülzsch K, Rinder H, Trèek J, Bader L, Wilhelm U, Heesemann J 2002. "Staphylococcus pettenkoferi", a novel staphylococcal species isolated from clinical specimens. Diag Microbiol Infect Dis 43: 175-182.

Vuong C, Otto M 2002. Staphylococcus epidermidis infections. Microb Infect 4: 481-489. 